\title{
Erratum: Atomic cluster expansion for accurate and transferable interatomic potentials [Phys. Rev. B 99, 014104 (2019)]
}

\author{
Ralf Drautz $(\mathbb{0}$
}

Q (Received 11 June 2019; published 23 December 2019)

DOI: $10.1103 /$ PhysRevB.100.249901

In Eq. (C6), the relative weight of the different $J$ couplings was omitted. The symbol $\left[\begin{array}{cccc}j_{1} & j_{2} & j_{3} & j_{4} \\ m_{1} & m_{2} & m_{3} & m_{4}\end{array}\right]$ depends on the relative contribution of the different couplings $J$. The correct form of Eq. (C6) reads

$$
\left[\begin{array}{cccc}
j_{1} & j_{2} & j_{3} & j_{4} \\
m_{1} & m_{2} & m_{3} & m_{4}
\end{array}\right]=\sum_{J} c_{J} \sum_{M=-J}^{J}(-1)^{M} C_{J j_{1} j_{2}}^{M m_{1} m_{2}} C_{J j_{3} j_{4}}^{-M m_{3} m_{4}},
$$

with constants $c_{J}$ that determine the relative contributions of the different couplings $J$ and $\sum_{J} c_{J}^{2}=1$. The coefficients $c_{J}$, in general, differ for different basis functions indexed by $n_{1} n_{2} n_{3} n_{4} j_{1} j_{2} j_{3} j_{4}$.

In analogy, the correct expression for Eq. (C8) is given by

$$
\left[\begin{array}{ccccc}
j_{1} & j_{2} & j_{3} & j_{4} & j_{5} \\
m_{1} & m_{2} & m_{3} & m_{4} & m_{5}
\end{array}\right]=\sum_{J_{1} J_{2}} c_{J_{1} J_{2}} \sum_{M_{1} M_{2}}(-1)^{M_{2}} C_{J_{1} j_{1} j_{2}}^{M_{1} m_{1} m_{2}} C_{J_{2} J_{1} j_{3}}^{M_{2} M_{1} m_{3}} C_{J_{2} j_{4} j_{5}}^{-M_{2} m_{4} m_{5}},
$$

with $\sum_{J_{1} J_{2}} c_{J_{1} J_{2}}^{2}=1$.

None of the conclusions or results of the paper are affected by these typing errors.

I am indebted to Dr. A. Shapeev from Skoltech, Russia for pointing out the mistake in Eq. (C6). 